\title{
Selective mode suppression in microstrip differential lines by means of electric-LC (ELC) and magnetic-LC (MLC) resonators
}

\author{
J. Naqui, M. Durán-Sindreu, and F. Martín \\ GEMMA/CIMITEC, Departament d'Enginyeria Electrònica, \\ Universitat Autònoma de Barcelona, 08193-BELLATERRA (Barcelona), Spain \\ *corresponding author: Ferran.Martin@uab.cat
}

\begin{abstract}
In this paper, it is demonstrated that the so-called electricLC (ELC) resonators, and their dual counterparts, the magnetic-LC (MLC) resonators, are useful for the selective suppression of either the differential or the common mode in microstrip differential lines. The key point to mode suppression is the alignment of the resonator with the electric (differential mode) or magnetic (common mode) wall of the line. It is shown that by simply rotating the resonators $90^{\circ}$ we can selectively choose the suppressed mode in the vicinity of the resonator's fundamental resonance frequency. The theory is validated through fullwave electromagnetic simulation, the lumped element equivalent circuit models of the proposed structures and experimental data.
\end{abstract}

\section{Introduction}

Split-ring resonators (SRRs) [1] and their complementary counterparts (CSRRs) [2] (Fig. 1) have been extensively used for the implementation of metamaterials and many devices based on them [3]. One of the applications of these metamaterial resonators is the selective mode suppression in multimode transmission lines. Specifically, SRRs etched in the back substrate side of a CPW with their symmetry plane ( $x-z$ plane) aligned with the symmetry plane of the line, are useful to suppress the parasitic slot mode of the line at their fundamental resonance, whereas they are transparent for the fundamental CPW (common) mode [4] [Fig. 2(a)]. On the other hand, CSRRs have been applied to suppress the common mode in microstrip differential lines and balanced circuits [5]. In these applications, the CSRRs must be etched in the ground plane with their symmetry plane aligned with the symmetry plane of the differential line [Fig. 2(b)].

The key aspect to suppress either the differential or the common mode, keeping the other mode unaltered (or almost unaltered), is the perfect alignment of electric or magnetic walls between the line and the resonator. Thus, for the CPW loaded with symmetrically etched SRRs, the electric wall of the SRR at their symmetry plane is responsible for the slot mode suppression, since the $\mathrm{CPW}$ exhibits an electric wall at the symmetry plane for this mode. For a microstrip differential line, the symmetry plane is a magnetic wall for the common mode. Therefore, by symmetrically etching a CSRR in the ground plane, where the symmetry plane is also a magnetic wall, the common mode is suppressed in the vicinity of the fundamental CSRR resonance, whereas the differential mode is kept unaltered.

SRRs/CSRRs exhibit bianisotropy [3,6,7], which means that they can be excited by means of a uniform axial timevarying magnetic/electric field, and/or through an electric/magnetic field orthogonally applied to the symmetry plane of the particle. When these particles (SRRs or CSRRs) are loading elements of a transmission line, it is clear that the driving fields generated by the line are not uniform. Nevertheless, it has been demonstrated that both CPW and microstrip lines loaded with symmetrically etched SRRs and CSRRs, respectively, exhibit mixed coupling between the line and the resonators for those line modes that are suppressed [8]. (a)

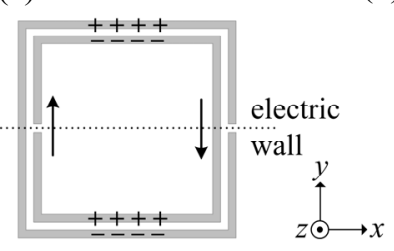

(b)

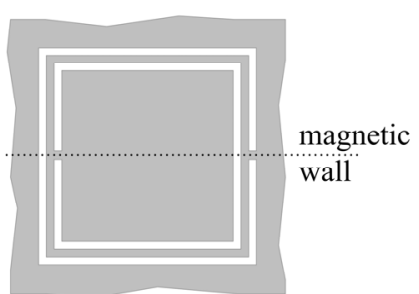

Figure 1: Typical topology of an SRR (a) and a CSRR (b). A sketch of the currents and the distribution of charges for the SRR at the fundamental resonance is also indicated. Notice that the symmetry plane is an electric wall. From the Babinet principle, it follows that the CSRR exhibits a magnetic wall at the symmetry plane.
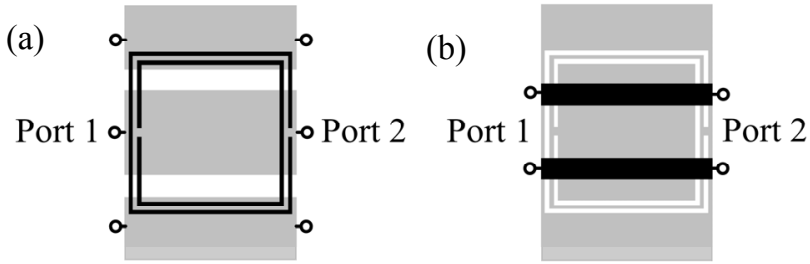

Figure 2: CPW loaded with a symmetrically etched SRR (a) and microstrip differential line loaded with a symmetrically etched CSRR (b). 
(a)

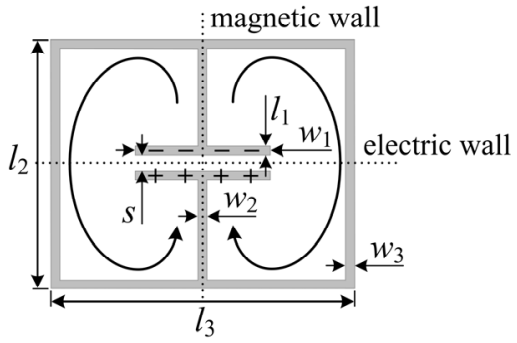

(b)

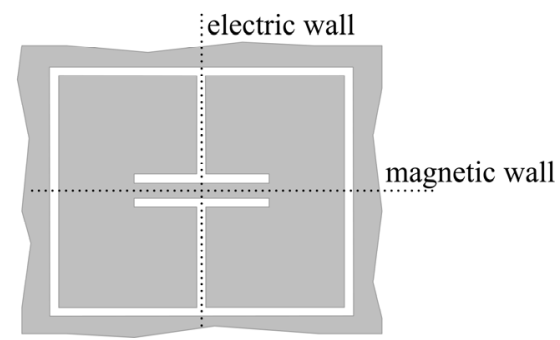

Figure 3: Typical topology of an ELC (a) and an MLC (b) resonator. The nature of the symmetry planes at the fundamental resonance is indicated, as well as a sketch of the currents and the distribution of charges for the ELC.

In this paper, alternative resonators are considered, namely, the so-called electric-LC resonator, ELC [9], and its complementary particle, the magnetic-LC resonator, MLC (see Fig. 3). Such resonators are bisymmetric, one of the symmetry planes being a magnetic wall and the other one being an electric wall at the fundamental resonance. Moreover, the resonators are non-bianisotropic because of the inversion symmetry with regard to its center. The selective mode suppression with ELC and MLC resonators in microstrip differential lines is discussed on the basis of symmetry properties. The theory is validated through electromagnetic simulation, circuit simulation of the lumped element equivalent circuit models, and experiment.

\section{ELC and MLC resonators}

The ELC resonator was proposed by Schurig et al. for the implementation of resonant-type negative permittivity metamaterials, as an alternative to the well known wire media, described by the Drude model [9]. The ELC resonator consists of two closed inductive loops connected to a common capacitor. At the fundamental resonance, the instantaneous current is clockwise in one loop and counterclockwise in the other loop [see Fig. 3(a)], thus giving rise to a net displacement current in the central capacitor. Therefore, at the fundamental resonance, the magnetic moment associated with currents in both loops cancels and the particle cannot be excited by a uniform time-varying magnetic field applied in the axial direction (this is the main driven mechanism in SRRs). However, there is still an electric dipole moment associated with the capacitor. Thus, the ELC resonator can be electrically driven (or coupled) by means of an electric field applied in the particle plane in the direction orthogonal to the electric wall. Notice that due to charge and current symmetry, the vertical symmetry plane in Fig. 3(a) is a magnetic wall for the fundamental resonance.

For the MLC, we can invoke the Babinet principle and conclude that this particle also exhibits electric and magnetic walls, rotated $90^{\circ}$ as compared to those of the ELC. In other words, the particle cannot be excited by means of a uniform axial time-varying electric field (as a CSRR is driven), but it can be driven through a magnetic field orthogonally applied to the magnetic wall.

\section{Microstrip differential lines loaded with ELCs and MLCs}

As it was discussed before [5], if a symmetric transmission line is loaded with a symmetric resonator with both symmetry planes aligned, and the symmetry planes are of the same nature (electric or magnetic walls), signal propagation in the vicinity of the fundamental resonance is inhibited (i.e., a transmission zero appears). However, the line is transparent if such symmetry planes are distinct. In particular, if we consider a microstrip differential line loaded with an ELC or an MLC, there are 4 cases of interest for selective mode suppression, depicted in Figs. 4 and 5. In Figs. 4(a) and (b), the differential line is loaded with an ELC etched on the upper side of the substrate, with a relative orientation of $90^{\circ}$ between the loading elements. According to these orientations, it is expected that the structure of Fig. 4(a) is transparent for the differential mode and produces a notch for the common mode, contrarily to Fig. 4(b).
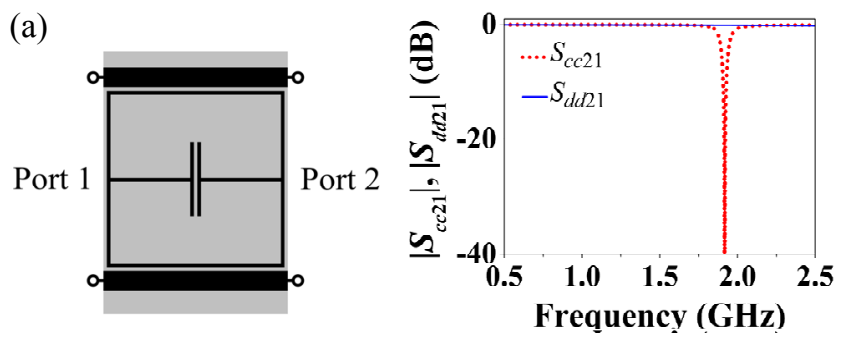

(b)

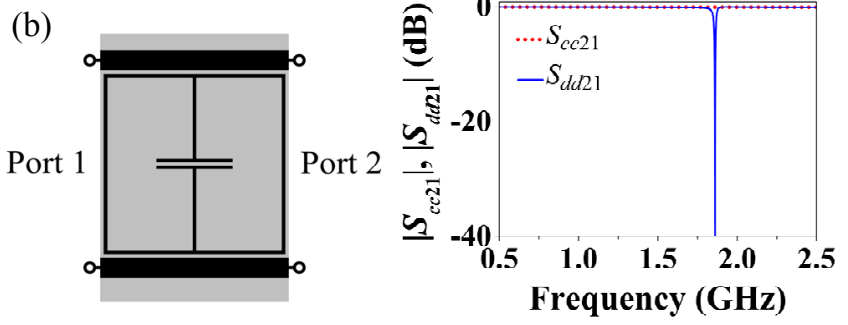

Figure 4: Microstrip differential line loaded with an ELC with the magnetic (a) and electric (b) wall aligned with the symmetry plane of the line. The substrate is Rogers RO3010 with thickness $h=1.27 \mathrm{~mm}$ and dielectric constant $\varepsilon_{r}=10.2$. The line dimensions are: line width $W=1.1 \mathrm{~mm}$ and line separation $S=10.4 \mathrm{~mm}$, which corresponds to a $50 \Omega$ even mode (common mode related) and odd mode (differential mode related) characteristic impedance. The ELC dimensions are: $w_{1}=4 \mathrm{~mm}, w_{2}=w_{3}=l_{1}=s=0.2 \mathrm{~mm}, l_{2}=l_{3}=10 \mathrm{~mm}$. 
(a)
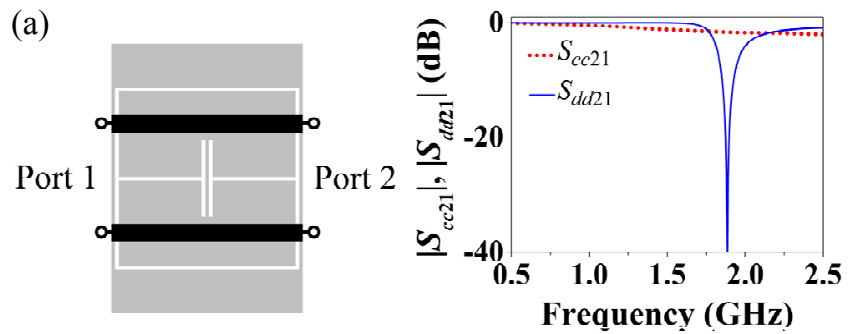

(b)

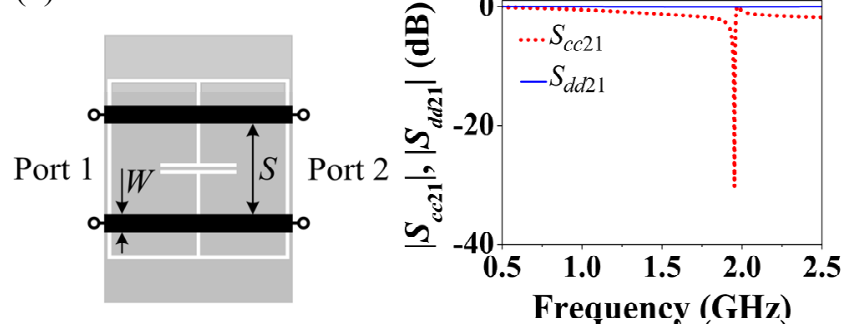

Figure 5: Microstrip differential line loaded with an MLC with the electric (a) and magnetic (b) wall aligned with the line. The substrate is Rogers RO3010 with thickness $h=1.27 \mathrm{~mm}$ and dielectric constant $\varepsilon_{r}=10.2$. The line dimensions are: $W=1 \mathrm{~mm}$ and $S=5 \mathrm{~mm}$, corresponding to a $50 \Omega$ even mode and odd mode characteristic impedance without the MLC etching. The MLC dimensions are: $w_{1}=4 \mathrm{~mm}$, $w_{2}=w_{3}=l_{1}=s=0.2 \mathrm{~mm}$, and $l_{2}=l_{3}=10 \mathrm{~mm}$.

In Figs. 5(a) and 5(b), the differential line is loaded with an MLC resonator etched in the ground plane. In this case the situation is reversed since the electric and magnetic walls of ELCs and MLCs are interchanged. The reflection and the transmission coefficients for the differential $\left(S_{d d 11}\right.$ and $\left.S_{d d 21}\right)$ and common $\left(S_{c c 11}\right.$ and $\left.S_{c c 21}\right)$ modes of the structures of Figs. 4 and 5 have been inferred by means of the Agilent Momentum commercial software. The transmission coefficient results (also depicted in Figs. 4 and 5) validate the selective mode suppression achievable in these differential lines by merely rotating the resonators $90^{\circ}$.

It is important to emphasize that the driving fields of the particles are not uniform. Indeed, in the configuration of Fig. 4(a), the ELC cannot be excited by the electric field generated by the differential line in the common mode. However, for this mode, the magnetic fields generated by the differential line have contra-directional axial components with regard to the midplane, sufficient to excite the particle. Therefore, the ELC resonator is excited by means of the magnetic field generated by the line under common mode operation. Similarly, in the configuration of Fig. 5(a), the MLC is excited under differential mode operation thanks to the electric field generated by the pair of lines, which is able to induce an electric dipole moment and a net current flow in the ground plane, across the symmetry plane of the differential line (or across the electric wall of the MLC). Thus, the terminology of these particles does not obey to the nature of the driving fields in the considered configurations (the reason is that the fields are not uniform). However, the original nomenclature, given by Schurig et al. [9] to the resonator of Fig. 3(a), i.e. ELC, is preserved. For coherence, the resonator of Fig. 3(b) is called MLC particle by the authors.

\section{Lumped element equivalent circuit models}

Let us now consider the lumped element equivalent circuit models of the structures of Figs. 4 and 5. In Fig. 4(a), the ELC can only by excited by the magnetic field generated by the line (common mode), but not by the electric field, due to the particle orientation. Similarly, for the MLC of Fig. 5(a), excitation under differential mode operation is only possible through the electric field generated by the line. In Figs. 4(b) and 5(b), particle excitation under differential and common mode, respectively, is more complex, since both electric and magnetic coupling between the line and the resonator must be considered. Let us thus start with the lumped element equivalent circuit models of the structures of Figs. 4(a) and 5(a), where mixed coupling is not present.

The model of the ELC-loaded differential line of Fig. 4(a) is depicted in Fig. 6(a). The electric and magnetic coupling between the pair of lines has been neglected due to the significant separation between the individual lines (necessary to accommodate the ELC). The per-section inductance and capacitance of the individual lines are modeled by $L$ and $C$, respectively. The ELC resonator is modeled by the inductances $L_{e}$ and the capacitance $C_{e}$, and the magnetic coupling between the lines and the resonator is accounted for through the mutual inductances $M$. The equivalent circuit models for even and odd mode (common and differential mode related, respectively) excitation are depicted in Figs. 6(b) and (c). For the common mode, the symmetry plane is an open circuit, and the model is identical to that of an SRR-loaded line, giving a notch in the transmission coefficient at resonance $[10,11]$. For the differential mode, the symmetry plane is a short circuit and the ELC no longer plays an active role. Therefore, the line is transparent for this mode. For the ELC-loaded line of Fig. 7, we have extracted the circuit parameters from the electromagnetic simulation of the frequency response for the common mode following the procedure explained in [12]. The agreement between circuit and electromagnetic simulations is remarkable. Notice that the topology of the ELC of Fig. 7 has been modified as compared with that of Fig. 4 in order to reduce the electrical size of the particle at resonance (under these conditions the lumped element circuit model is valid in a wider frequency range).

The model of the MLC-loaded differential line of Fig. 5(a) is depicted in Fig. 8(a). The per-section line inductance and capacitance are modeled, respectively, by $L$ and $C$ (again, the coupling between the line pair is not significant). However, notice that actually $L$ is the line inductance with the presence of the MLC and $C$ acts as the coupling capacitance between the lines and the MLC. The resonator is modeled by the inductance $L_{m}$ and the capacitances $C_{m}$. Finally, the particle is capacitively connected to the ground plane through the slot ring capacitance modeled by $C_{g}$. The equivalent common and differential mode models are depicted in Figs. 8(b) and 8(c), respectively. For the common mode, the resonator is opened, and the resulting 
model is that of a transmission line with modified shunt capacitance. Thus, some attenuation can occur as can be seen in Fig. 5(a), as a consequence of the MLC etching (i.e., a defected ground structure) that disturbs the return current flowing on the ground plane modifying the line parameters [5]. On the other hand, for the differential mode, there is a short in the symmetry plane, and the circuit model is identical to that of a CSRR-loaded microstrip line, providing a notch in the transmission coefficient for such mode at resonance. We have considered the MLC-loaded line of Fig. 9, and we have extracted the circuit parameters for the differential mode from the electromagnetic simulation of the frequency response for this mode as reported in [13]. The agreement between circuit and electromagnetic simulations is also good.

Let us now focus on the lumped element equivalent circuit models that result by rotating the ELC and the MLC $90^{\circ}$, that is, the models describing the structures of Figs. 4(b) and 5(b), where both electric and magnetic coupling must be considered. For the structure of Fig. 4(b), the circuit model is that depicted in Fig. 10(a). In this circuit, the magnetic coupling's sign is provided by the dot convention to avoid any ambiguity. The resonator has been rotated, and the coupling capacitances, $C_{a}$, have been added to account for electric coupling. Indeed, since the symmetry plane in the configuration of the structure of Fig. 4(b) is an electric wall for the differential mode, electric coupling is the main driving mechanism of the ELC for this mode (the magnetic coupling is not able to induce resonant currents by itself). The circuit models for the common and differential modes are depicted in Figs. 10(b) and 10(c), respectively, where it is clear that the common mode is not able to excite the particle and the structure is transparent for this mode. Conversely, the differential mode produces a notch in the

(a)

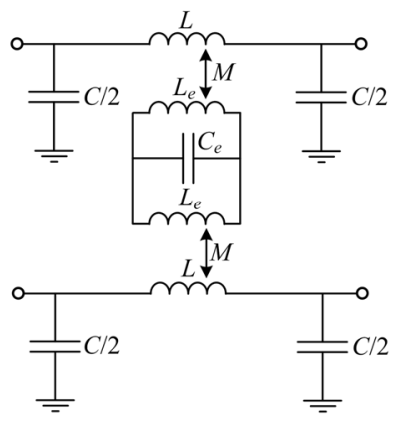

(b)

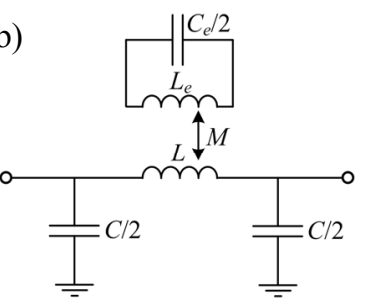

(c)

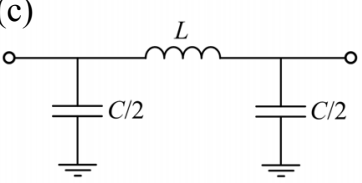

Figure 6: (a) Lumped element equivalent circuit model of the ELC-loaded differential line of Fig. 4(a); (b) circuit model for the even mode; (c) circuit model for the odd mode.

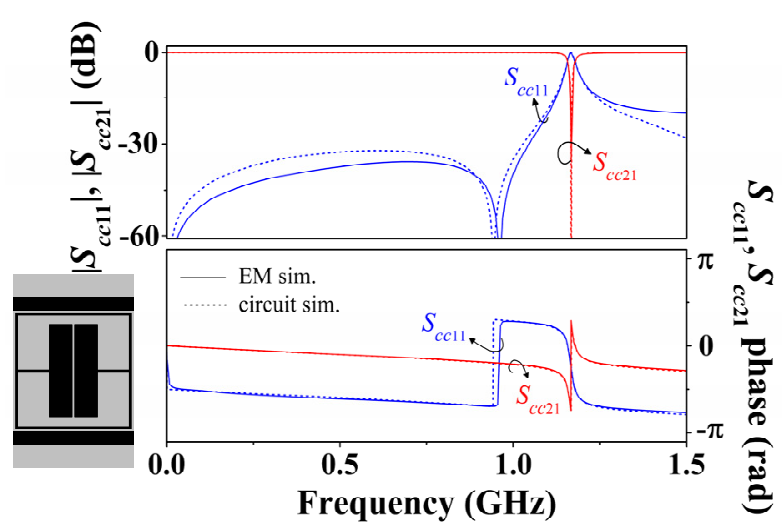

Figure 7: Frequency response (common mode) for the structure of Fig. 4(a) with $l_{1}=2 \mathrm{~mm}$ and $w_{1}=8 \mathrm{~mm}$ and for its circuit model of Fig. 6(b). The circuit parameters are: $L=4.57 \mathrm{nH}, \quad C=2.2 \mathrm{pF}, \quad L_{e}=4.57 \mathrm{nH}$, $C_{e}=8.14 \mathrm{pF}$, and $M=1 \mathrm{nH}$.

(a)

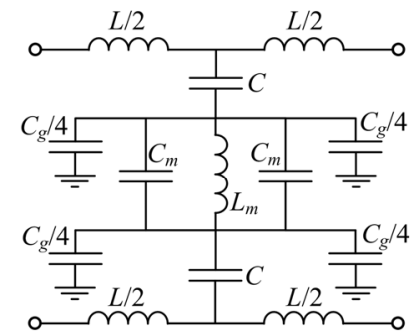

(b)
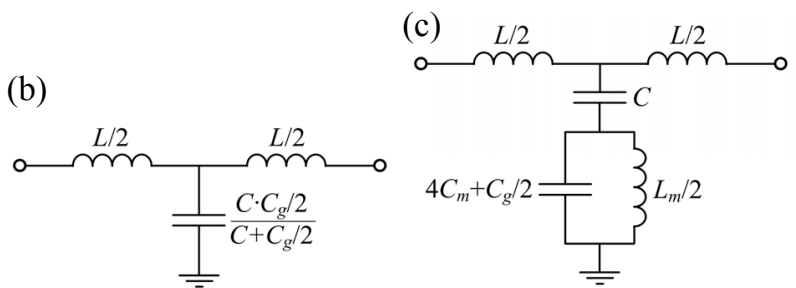

Figure 8: (a) Lumped element equivalent circuit model of the MLC-loaded differential line of Fig. 5(a); (b) circuit model for the even mode; (c) circuit model for the odd mode.

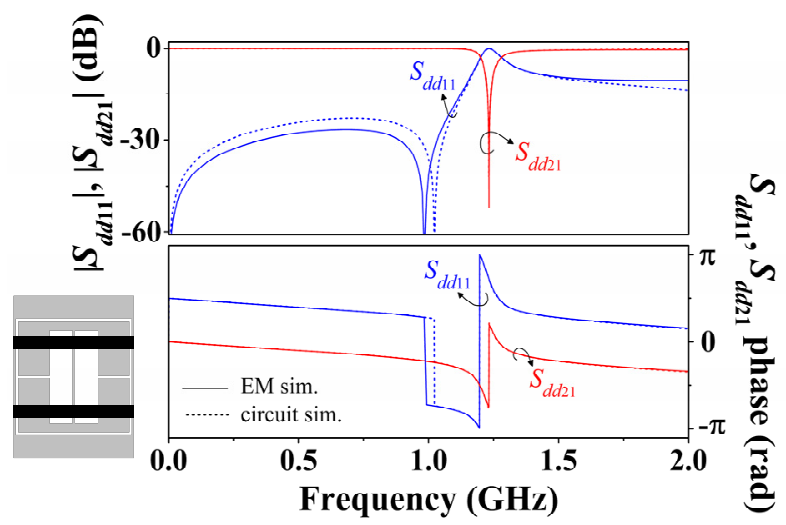

Figure 9: Frequency response (differential mode) for the structure of Fig. 5(a) with $l_{1}=2 \mathrm{~mm}$ and $w_{1}=8 \mathrm{~mm}$ and for its circuit model of Fig. 8(c). The circuit parameters are: $L=6.07 \mathrm{nH}, C=1.53 \mathrm{pF}, 4 C_{m}+C_{g} / 2=7.47 \mathrm{pF}$, and $L_{m}=3.71 \mathrm{nH}$. 
transmission coefficient, as the equivalent circuit model of such mode confirms. In order to match the frequency response for the circuit model (differential mode) to that inferred from the electromagnetic simulation, we have considered the structure of Fig. 11 and we have tuned the coupling capacitance, readjusting slightly the rest of parameters corresponding to Fig. 7 (the geometries of the differential line and the ELC are identical). As can be seen, the agreement is reasonably good.

(a)

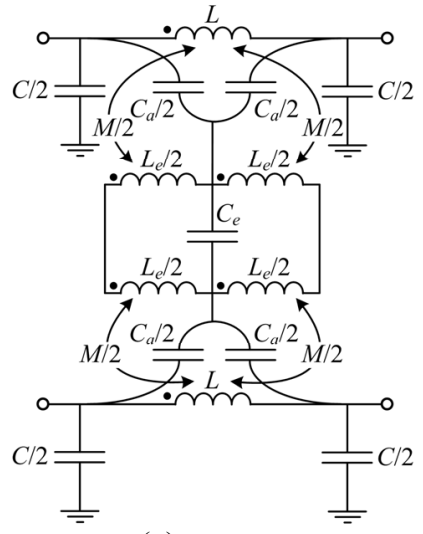

(c)

(b)

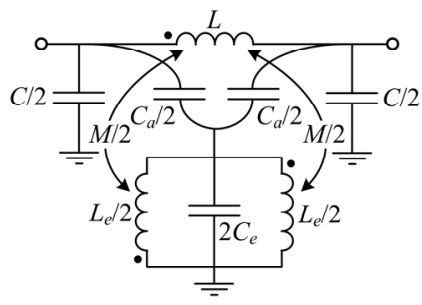

Figure 10: (a) Lumped element equivalent circuit model of the ELC-loaded differential line of Fig. 4(b); (b) circuit model for the even mode; (c) circuit model for the odd mode.

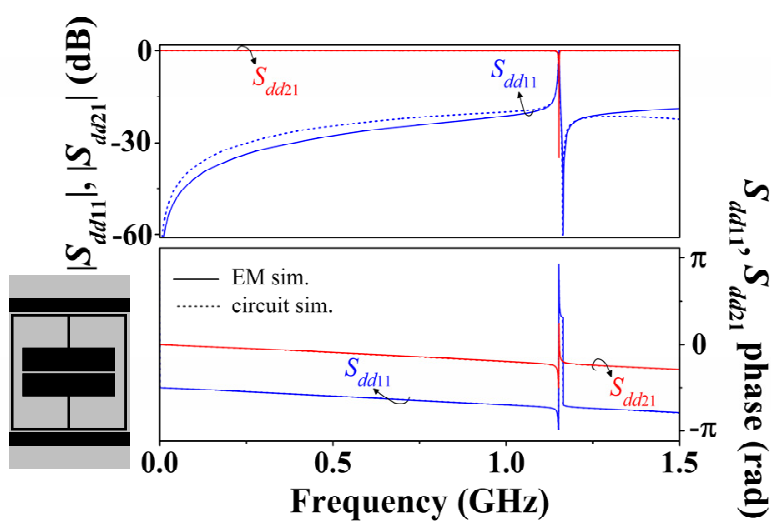

Figure 11: Frequency response (differential mode) for the structure shown in Fig. 4(b) with $l_{1}=2 \mathrm{~mm}$ and $w_{1}=8 \mathrm{~mm}$ and for its circuit model in Fig. 10(c). The circuit parameters are: $L=4.09 \mathrm{nH}, C=1.88 \mathrm{pF}$, $L_{e}=4.62 \mathrm{nH}, \quad C_{e}=8.04 \mathrm{pF}, \quad M=1.12 \mathrm{nH}, \quad$ and $C_{a}=0.44 \mathrm{pF}$.

The MLC-loaded differential line of Fig. 5(b) is modeled by the circuit of Fig. 12(a). This is obtained by rotating the particle and splitting the coupling capacitances. Moreover, magnetic coupling has been introduced and is accounted for by the mutual inductances $M$. The equivalent circuit models for the common and differential modes, which are shown respectively in Figs. 12(b) and 12(c), indicate that the structure is transparent for the differential mode, whereas a notch appears for the common mode. For the latter mode, the magnetic coupling to the strip connecting the two inner halves of the MLC is absolutely necessary to excite the particle. Otherwise, resonance could not be driven exclusively through the electric coupling. Notice also that, by applying the magnetic wall concept, the resulting mutual inductance in Fig. 12(b) is twice that considered in Fig. 12(a). Conversely, an electric wall in Fig. 10(a) does not scale the mutual inductance, as can be appreciated in Fig. 10(c). Again, the electromagnetic simulation for the common mode is compared to the circuit simulation for such mode (Fig. 13), and the agreement is also good, thus confirming the validity of the proposed model.

(a)
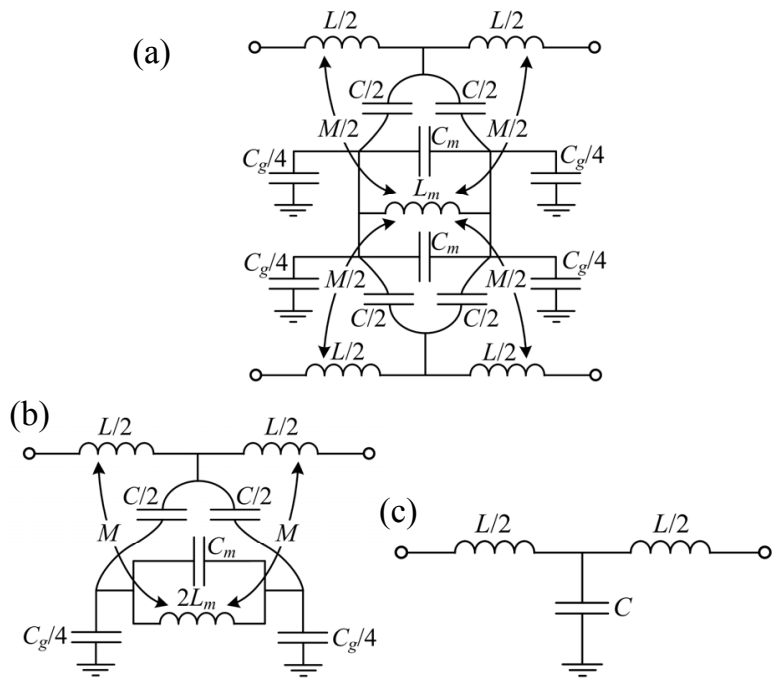

Figure 12: (a) Lumped element equivalent circuit model of the MLC-loaded differential line of Fig. 5(b); (b) circuit model for the even mode; (c) circuit model for the odd mode.

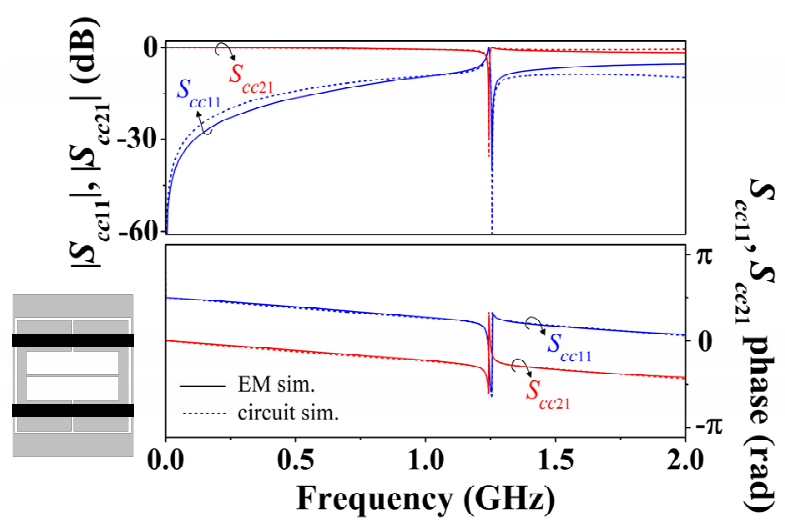

Figure 13: Frequency response (common mode) for the structure shown in Fig. 5(b) with $l_{1}=2 \mathrm{~mm}$ and $w_{1}=8 \mathrm{~mm}$ and for its circuit model in Fig. 12(b). The circuit parameters are: $L=9.3 \mathrm{nH}, C=1.53 \mathrm{pF}$, $L_{m}=3.64 \mathrm{nH}$, and $C_{m}=0.93 \mathrm{pF}, C_{g}=7.5 \mathrm{pF}$, and $M=0.5 \mathrm{nH}$. 
Finally, it is worth to highlight that all the proposed structures are symmetric with regard to the ports (i.e., $S_{c c 11}=S_{c c 22}$ and $S_{d d 11}=S_{d d 22}$ ), and their corresponding circuits as well.

\section{Applications}

The proposed structures can be useful for common mode suppression in differential lines and balanced circuits [5], for differential notch/stopband filters, or for the implementation of sensors based on symmetry properties [14]. Specifically, with a circular structure based on the configurations of Fig. 4, it is possible to implement a rotation sensor in microstrip technology similar to that based on the CPW structure reported in [14]. On the other hand, as compared with the structures used in [5], based on CSRRs, the structure of Fig. 4(a) is able to suppress the common mode keeping the ground plane unaltered. However, this solution seems to be not so suitable for common mode suppression because of its inherent narrow-band rejection. On the contrary, a narrow balanced stopband filter with low insertion loss in the transmission bands can be useful in order to suppress undesired differential signals. To this end, we have designed an order-3 balanced stopband filter based on the structure of Fig. 5(a). The filter is composed of three cascaded MLCs with slightly different longitudinal dimensions to widen the stopband. A photograph of the fabricated filter and the frequency response are shown in Fig. 14. The measured response exhibits a rejection of more than $20 \mathrm{~dB}$ for differential signals over a fractional bandwidth of $14.3 \%$ centered at $1.3 \mathrm{GHz}$.

\section{Conclusions}

In conclusion, it has been demonstrated that the differential and common modes in microstrip differential lines can be selectively suppressed by loading the lines with either ELC or MLC bisymmetric resonators. Key to this selective mode suppression at the fundamental resonance frequency of the particles is the presence of a magnetic and an electric wall, orthogonally oriented, in these resonators. Hence, it is possible to either suppress the common or the differential mode by simply rotating the considered particle (ELC or MLC) $90^{\circ}$. We have proposed lumped element circuit models for microstrip differential lines loaded with both resonators for the two considered orientations, and the agreement between the frequency responses inferred from circuit and electromagnetic simulations is good. Finally, potential applications have been highlighted, and a differential stopband filter has been presented.

\section{Acknowledgements}

This work has been supported by MICIIN-Spain (projects TEC2010-17512, CSD2008-00066, and TEC2011-13615E) and by AGAUR-Generalitat de Catalunya, through project 2009SGR-421. Jordi Naqui is also in debt to MINECO (Spain) for supporting his work through the FPU grant AP2010-0431. (a)
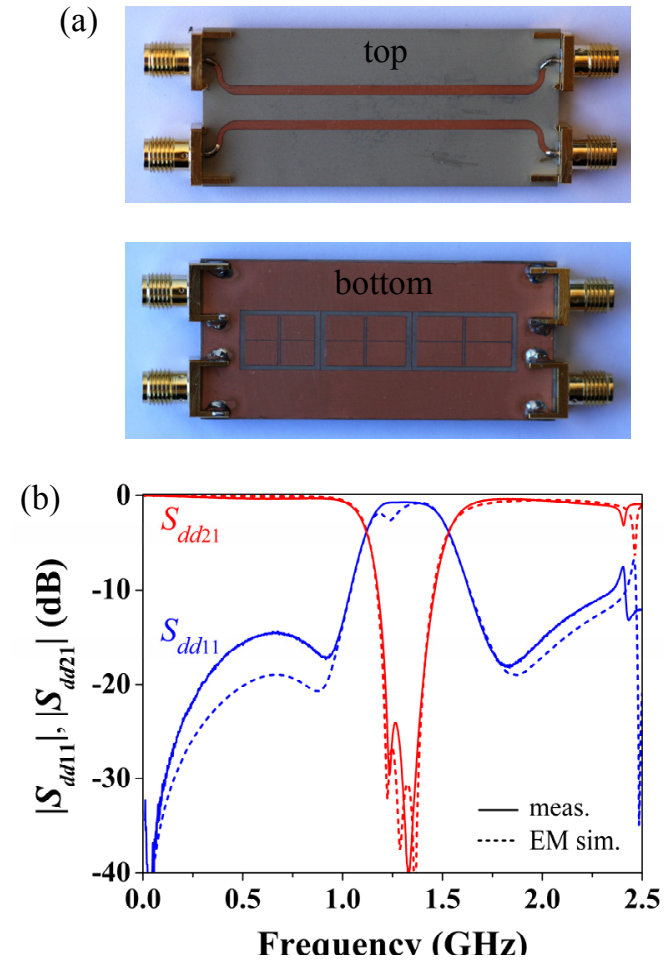

Figure 14: (a) Photograph and (b) differential mode frequency response for the designed differential stopband filter. The substrate is Rogers RO3010 with $h=1.27 \mathrm{~mm}, \varepsilon_{r}=10.2$, and loss tangent $\tan (\delta)=0.0023$. Line dimensions are: $W=1.6 \mathrm{~mm}$ and $S=5 \mathrm{~mm}$ (not corresponding to a $50 \Omega$ odd mode impedance), designed to mitigate the passband insertion loss due to the presence of the resonators. MLC dimensions are: $w_{1}=8 \mathrm{~mm}, w_{2}=l_{1}=s=0.2 \mathrm{~mm}, w_{3}=0.8 \mathrm{~mm}$, $l_{2}=[13.2,15.1,17.6] \mathrm{mm}$, and $l_{3}=10 \mathrm{~mm}$. The interresonator distance is $0.2 \mathrm{~mm}$.

\section{References}

[1] J.B. Pendry, "Magnetism from conductors and enhanced nonlinear phenomena", IEEE Trans. Microw. Theory Tech., vol. 47, pp. 2075-2084, Nov.1999.

[2] F. Falcone, T. Lopetegi, J. D. Baena, R. Marqués, F. Martín, and M. Sorolla, "Effective negative- $\varepsilon$ stop-band microstrip lines based on complementary split ring resonators," IEEE Microwave and Wireless Components Letters, vol. 14, pp. 280-282, June 2004.

[3] R. Marqués, F. Martín, M. Sorolla, Metamaterials with negative parameters: theory, design and microwave applications, John Wiley \& Sons Inc., USA, 2008.

[4] J. Naqui, M. Durán-Sindreu, F. Martín, “Selective mode suppression in coplanar waveguides using metamaterial resonators", Appl. Phys. A - Materials Science and Processing, vol. 109, pp. 1053-1058, Dec. 2012.

[5] J. Naqui, A. Fernández-Prieto, M. Durán-Sindreu, F. Mesa, J. Martel, F. Medina, and F. Martín, "Common mode suppression in microstrip differential lines by means of complementary split ring resonators: theory and applications", IEEE Transactions on Microwave 
Theory and Techniques, vol. 60, pp. 3023-3034, Oct. 2012.

[6] R. Marques, F. Medina and R. Rafii-El-Idrissi, "Role of bi-anisotropy in negative permeability and left handed metamaterials", Phys. Rev. B, vol. 65, paper 144441, 2002.

[7] R. Marqués, F. Mesa, J. Martel and F. Medina, "Comparative analysis of edge and broadside couple split ring resonators for metamaterial design. Theory and Experiment", IEEE Trans. Ant. Propag., vol. 51, pp.2572-2582, Oct. 2003.

[8] J. Naqui, M. Durán-Sindreu, and F. Martín, "Modeling split ring resonator (SRR) and complementary split ring resonator (CSRR) loaded transmission lines exhibiting cross polarization effects", IEEE Antennas and Wireless Propagation Letters, vol. 12, pp. 178-181, 2013.

[9] D. Schurig, J. J. Mock, and D. R. Smith, "Electric-fieldcoupled resonators for negative permittivity metamaterials", Appl. Phys. Lett., vol. 88, paper 041109, 2006.

[10]F. Martín, F. Falcone, J. Bonache, R. Marqués and M. Sorolla, "Split ring resonator based left handed coplanar waveguide", Appl. Phys. Lett., vol. 83, pp. 4652-4654, December 2003.

[11]F. Aznar, J. Bonache and F. Martín, "Improved circuit model for left handed lines loaded with split ring resonators", Appl. Phys. Lett., vol. 92, paper 043512, February 2008.

[12]F. Aznar, M. Gil, J. Bonache, L. Jelinek, J. D. Baena, R. Marqués and F. Martín, "Characterization of miniaturized metamaterial resonators coupled to planar transmission lines through parameter extraction", $J$. Appl. Phys., vol. 104, pp. 114501, December 2008.

[13] J. Bonache, M. Gil, I. Gil, J. Garcia-García and F. Martín, "On the electrical characteristics of complementary metamaterial resonators", IEEE Microw. Wireless Compon. Lett., vol. 16, pp. 543.545, October 2006.

[14] J. Naqui, M. Durán-Sindreu, F. Martín, “Transmission Lines Loaded with Bisymmetric Resonators and Applications", IEEE MTT-S Int. Microwave Symp., June 2013, Seatle (USA). 